\title{
Regeneration of carbon nanotubes exhausted with dye reactive red 3BS using microwave irradiation
}

\author{
Jun Wang, Xianjia Peng*, Zhaokun Luan, Changwei Zhao \\ State Key Laboratory of Environmental Aquatic Chemistry, Research Center for Eco-Environmental Sciences, Chinese Academy of Sciences, \\ 18 Shuangqing Road, Beijing 100085, China
}

\section{A R T I C L E I N F O}

\section{Article history:}

Received 5 November 2009

Received in revised form

30 December 2009

Accepted 21 January 2010

Available online 28 January 2010

\section{Keywords:}

Carbon nanotubes

Regeneration

Microwave irradiation

Dye

\begin{abstract}
A B S T R A C T
Carbon nanotubes (CNTs) exhausted with dye reactive red 3BS were regenerated by microwave irradiation under $\mathrm{N}_{2}$ atmosphere. High regeneration efficiency was achieved and the regeneration efficiency reached $92.8 \%$ after four cycles regeneration. The decrease in adsorption capacity was suggested to be due to the deposition of decomposition residues in CNT pores, which blocked the carbon porosity and decreased the specific surface area.
\end{abstract}

(c) 2010 Elsevier B.V. All rights reserved.

\section{Introduction}

Carbon nanotubes (CNTs) are relatively new carbon materials used as adsorbents for removal of organic pollutants from water. Previous research has discovered that CNTs showed good removal capacity for organic pollutants. Peng et al. [1] found that CNTs are effective for removal of 1,2-dichlorobenzene from water in a wide $\mathrm{pH}$ range of 3-10. Lu et al. [2] investigated the removal of trihalomethanes by CNTs and found that purified CNTs have higher adsorption capacities for $\mathrm{CHCl}_{3}$ than activated carbon. The good performance of CNTs for removal of organic pollutants suggests that CNTs may have potential to be used as pollutant removal adsorbents. The applicability of adsorbents for removal of pollutants from water depends not only on the adsorption capacity, but also on the regeneration property and reusability. Until now, little study has been done on the regeneration of CNTs exhausted with organic pollutants.

As for the regeneration of carbon exhausted with organic pollutants, there are several methods, such as thermal treatment [3], surfactant based desorption [4-5], chemical oxidation [6-8], electrochemical oxidation [9] and microwave (MW) irradiation. Among them, MW irradiation has attracted attention due to its advantages such as molecular-level heating, fast regeneration rate and energy-saving [10]. Previous study has employed MW irradiation

\footnotetext{
* Corresponding author. Tel.: +86 10 62849198; fax: +86 1062849198.

E-mail address: xjpeng@rcees.ac.cn (X. Peng).
}

for regeneration of activated carbon exhausted with organic pollutants and found that high regeneration efficiency can be achieved. It seems interesting to employ MW irradiation for regeneration of CNTs exhausted with organic pollutants. In this investigation, CNTs exhausted with dye reactive red 3BS was regenerated using MW irradiation.

\section{Materials and methods}

\subsection{Materials}

Multi-walled CNTs with diameter of $60-80 \mathrm{~nm}$, length of 5-15 $\mu \mathrm{m}$ and purity of $95 \%$ were commercially obtained and used in this study.

\subsection{Regeneration methods}

Before each cycle of regeneration, CNTs were saturated by adding $1 \mathrm{~g}$ CNTs into $1 \mathrm{~L}$ of $200 \mathrm{mg} \mathrm{L}^{-1}$ dye reactive red 3BS solution and stirred for $12 \mathrm{~h}$. Our previous study has shown that under such condition, CNTs are saturated. Then CNTs were separated from water by filtration, dried at $100^{\circ} \mathrm{C}$ for $6 \mathrm{~h}$ and milled (particle size $<0.15 \mathrm{~mm}$ ). A domestic MW oven with frequency of $2450 \mathrm{MHz}$ was modified according to Liu et al. [11] for the regeneration. $1 \mathrm{~g}$ exhausted CNTs were put into the vertical quartz tube and regenerated under $\mathrm{N}_{2}$ atmosphere for 15 min with $\mathrm{MW}$ power of $500 \mathrm{~W}$. After the regeneration, the sample was cooled under $\mathrm{N}_{2}$ atmosphere to room temperature. The saturation-regeneration process 


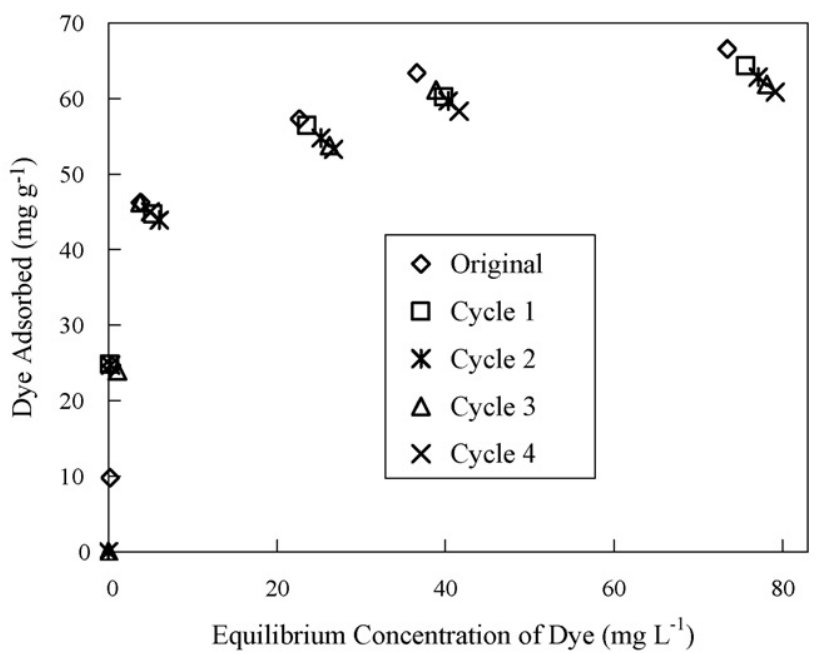

Fig. 1. Adsorption isotherms of original CNTs and regenerated CNTs.

has been carried out for four cycles and after each cycle, the adsorption capacity of the regenerated CNTs were evaluated by measuring the adsorption isotherm at temperature of $25^{\circ} \mathrm{C}$ and $\mathrm{pH}$ of 7.1.

\subsection{Textural characteristics}

Textural characteristics of the regenerated CNTs were determined by $\mathrm{N}_{2}$ adsorption isotherms on a Quantachrome model QuatraSorb SI surface area and porosimetry, Raman spectra on a Jobin Yvon model HR800 Raman spectrometer using $532 \mathrm{~nm}$ laser excitation and transmission electron microscopy (TEM) observation carried out on a FEI model TECNAI F30 field emission transmission electron microscope. Specific surface area (SSA) and pore volume (PV) were determined according to the $\mathrm{N}_{2}$ adsorption isotherms.

\section{Results and discussion}

The isotherms of original CNTs and regenerated CNTs were shown in Fig. 1. It can be seen that, after each cycle of regeneration, the removal of dye by CNTs only decreased slightly. The adsorption isotherms were fitted to Langmuir isotherm model and the adsorption capacity of original and regenerated CNTs were obtained and shown in Table 1. Adsorption capacity of original CNTs was $69.1 \mathrm{mg} \mathrm{g}^{-1}$ and after four cycles of regeneration, the capacity is $64.1 \mathrm{mg} \mathrm{g}^{-1}$. Adsorption capacity of CNTs after four cycles of regeneration is $92.8 \%$ of that of the original CNTs, which indicates that MW irradiation is rather effective for the regeneration of CNTs exhausted with dye reactive red 3BS. Otherwise, the measurement of CNT mass variation during MW treatment under $\mathrm{N}_{2}$ atmosphere showed no significant change of sample weight.

As for the regeneration of carbon exhausted with organic matters by MW irradiation, several processes may occur during the regeneration. Upon MW irradiation heating, gases coming from desorption of volatiles and the decomposition of organic matters adsorbed are evolved [10]. The release of the gases will modify the SSA and porosity of carbon, which will enhance the adsorption capacity of carbon. On the other hand, during the decomposition of organics, the partially decomposed organic matter will form coke

Table 1

Adsorption capacity of original CNTs and regenerated CNTs $\left(\mathrm{mg} \mathrm{g}^{-1}\right)$.

\begin{tabular}{lllll}
\hline Original & Cycle 1 & Cycle 2 & Cycle 3 & Cycle 4 \\
\hline 69.1 & 67.5 & 66.0 & 64.9 & 64.1 \\
\hline
\end{tabular}

Table 2

Specific surface area and pore volume of original CNTs and regenerated CNTs.

\begin{tabular}{llllll}
\hline & Original & Cycle 1 & Cycle 2 & Cycle 3 & Cycle 4 \\
\hline $\mathrm{SSA}\left(\mathrm{m}^{2} \mathrm{~g}^{-1}\right)$ & 127.6 & 116.3 & 109.0 & 100.3 & 94.7 \\
$\mathrm{PV}\left(\mathrm{ml} \mathrm{g}^{-1}\right)$ & 0.342 & 0.322 & 0.297 & 0.283 & 0.253 \\
\hline
\end{tabular}

deposits, which will block the porosity of the carbon and decrease the adsorption capacity [12].

SSA and PV characterization results were shown in Table 2. It can be seen that a slow and gradual fall in the SSA and porosity occurred after successive cycle of regeneration. This suggested that the MW irradiation process created residual deposits in the pores of CNTs, which blocked the porosity and reduced the SSA and PV.

TEM images of original CNTs and the CNTs after four cycles of regeneration were shown in Fig. 2. It was found that after the regen-
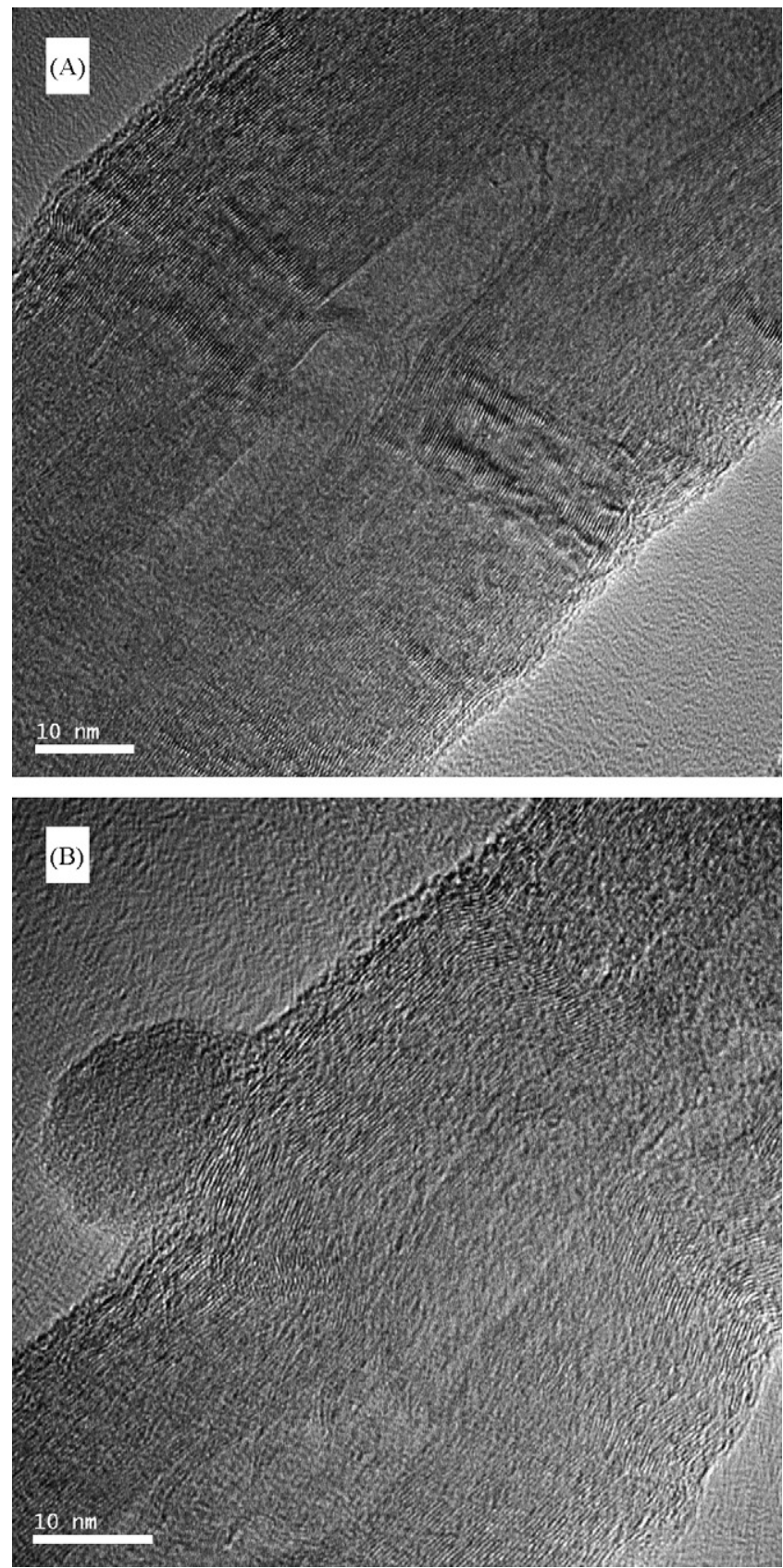

Fig. 2. TEM images of CNTs (A) and CNTs after four cycles of regeneration (B). 


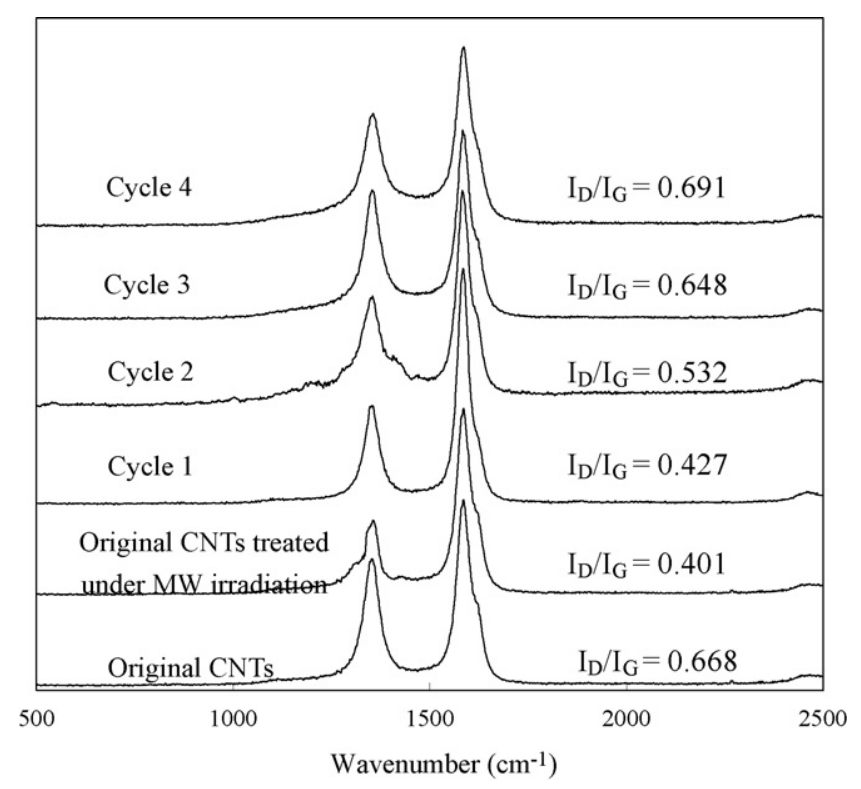

Fig. 3. Raman spectra of CNTs, CNTs treated with MW irradiation and exhausted CNTs regenerated under MW irradiation.

eration, some amorphous contamination is occasionally adsorbed to the outer walls of the CNTs, which indicated that decomposition of organic matter formed coke deposits on the outer surface of regenerated CNTs. Otherwise, it was found that after regeneration, CNTs have as good graphite structure as the original CNTs, with no obvious graphite-to-amorphous transformation of CNT outer walls during MW irradiation treatment, which indicated that the regeneration process did not damage CNT graphite structure.

In the typical Raman spectra of CNTs, the band centered at about $1580 \mathrm{~cm}^{-1}$ is noted as $\mathrm{G}$ band (Graphite band) and the band centered at about $1320 \mathrm{~cm}^{-1}$ is noted as D band (Disorder band). The $G$ band is related to the $\mathrm{C}-\mathrm{C}$ vibration of the carbon material with a $\mathrm{sp}^{2}$ orbital structure. The $\mathrm{D}$ band is contributed to the disorder-induced vibration of $\mathrm{C}-\mathrm{C}$ bond, which is indicative of the existence of amorphous carbon and graphitic impurities. The relative intensity ratio of $\mathrm{D}$ band to $\mathrm{G}$ band $\left(I_{\mathrm{D}} / I_{\mathrm{G}}\right)$ is known as an index to determine the CNT structure [13]. Raman spectra of the regenerated CNTs of each cycle were shown in Fig. 3. For comparison, spectra of original CNTs and original CNTs treated with MW irradiation were also included. The $I_{\mathrm{D}} / I_{\mathrm{G}}$ ratio decreased from 0.668 to 0.401 during the MW irradiation treatment of original CNTs, which indicated that under the conditions employed for the regeneration, CNTs are graphitized and a part of CNTs structure defects were removed. This was also confirmed by the fact that after one cycle of regeneration, the $I_{\mathrm{D}} / I_{\mathrm{G}}$ ratio of CNTs decreased (from 0.668 to 0.427 ). However, during the $1-4$ cycle of regeneration, the $I_{\mathrm{D}} / I_{\mathrm{G}}$ ratio gradually increased, which indicated that during each cycle of regeneration, residual deposits formed. This is consistent with the SSA and PV measure- ment and TEM observation results. The deposition of regeneration residues, which blocked the carbon porosity and decreased the SSA, will decrease the adsorption capacity. The deposition of regeneration residues in porosity of CNTs may mainly account for the slight decrease of adsorption capacity of CNTs after each cycle of regeneration.

\section{Conclusions}

MW irradiation under $\mathrm{N}_{2}$ atmosphere is effective for regeneration of CNTs exhausted with dye reactive red 3BS. High regeneration efficiency can be achieved and the regeneration efficiency reached $92.8 \%$ after four cycles regeneration. The slight decrease in adsorption capacity is maybe due to the deposition of decomposition residues in CNT pores, which blocked the carbon porosity and decreased the SSA.

\section{Acknowledgements}

This work was supported by National Natural Science Foundation of China (Grant No. 20607027) and the special fund from the State Key Laboratory of Environmental Aquatic Chemistry (Project No. 09Y10ESPCR).

\section{References}

[1] X.J. Peng, Y.H. Li, Z.K. Luan, Z.C. Di, H.Y. Wang, B.H. Tian, Z.P. Jia, Adsorption of 1,2-dichlorobenzene from water to carbon nanotubes, Chem. Phys. Lett. 376 (2003) 154-158.

[2] C.Y. Lu, Y.L. Chung, K.F. Chang, Adsorption of trihalomethanes from water with carbon nanotubes, Water Res. 39 (2005) 1183-1189.

[3] C. Moreno-Castill, J. Rivera-Utrill, J.P. Joly, M.V. López-Ramón, M.A. FerroGarcía, F. Carrasco-Marín, Thermal regeneration of an activated carbon exhausted with different substituted phenols, Carbon 33 (1995) 1417-1423.

[4] M.K. Purkait, S. DasGupta, S. De, Adsorption of eosin dye on activated carbon and its surfactant based desorption, J. Environ. Manage. 76 (2005) 135-142.

[5] M.K. Purkait, A. Maiti, S. DasGupta, S. De, Removal of Congo red using activated carbon and its regeneration, J. Hazard. Mater. 145 (2007) 287-295.

[6] L.C. Toledo, A.C.B. Silva, R. Augusti, R.M. Lago, Application of Fenton's reagent to regenerate activated carbon saturated with organochloro compounds, Chemosphere 50 (2003) 1049-1054.

[7] K. Okawa, K. Suzuki, T. Takeshita, K. Nakano, Regeneration of granular activated carbon with adsorbed trichloroethylene using wet peroxide oxidation, Water Res. 41 (2007) 1045-1051.

[8] R.V. Shende, V.V. Mahajani, Wet oxidative regeneration of activated carbon loaded with reactive dye, Waste Manage. 22 (2002) 73-83.

[9] N.W. Brown, E.P.L. Roberts, A.A. Garforth, R.A.W. Dryfe, Electrochemical regeneration of a carbon-based adsorbent loaded with crystal violet dye, Electrochim. Acta 49 (2004) 3269-3281.

[10] C.O. Ania, J.B. Parra, J.A. Menéndez, J.J. Pis, Microwave-assisted regeneration of activated carbons loaded with pharmaceuticals, Water Res. 41 (2007) 3299-3306.

[11] X.T. Liu, X. Quan, L.L. Bo, S. Chen, Y.Z. Zhao, Simultaneous pentachlorophenol decomposition and granular activated carbon regeneration assisted by microwave irradiation, Carbon 42 (2004) 415-422.

[12] C.O. Ania, J.A. Menéndez, J.B. Parra, J.J. Pis, Microwave-induced regeneration of activated carbons polluted with phenol. A comparison with conventional thermal regeneration, Carbon 42 (2004) 1383-1387.

[13] C.M. Chen, Y.M. Dai, J.G. Huang, J.M. Jehng, Intermetallic catalyst for carbon nanotubes (CNTs) growth by thermal chemical vapor deposition method, Carbon 44 (2006) 1808-1820. 